\title{
The Distribution Analysis for Extreme Returns of Nikkei 225 Index: Based on the Extreme Value Distribution of GEV and GL
}

\author{
Yuan An \\ China Institute for Actuarial Science \\ Central University of Finance and Economics \\ Beijing, 100081, China \\ Ernest Agyemang Duah \\ Department of Statistics and Actuarial Science \\ University of Waterloo, Waterloo. N2L 3G1, Canada
}

\begin{abstract}
This paper focuses on the problem of modelling extreme events in the financial market. The choice of the distribution that adequately models the extreme behavior of the financial time series. Extreme Value Theory outlines the framework for determining the best fit distribution for the data. The generalized extreme value distribution and the generalized Pareto distribution are the traditional distributions that most analysts resort to using. However, recent works have shown that the generalized logistic distribution can also capture the effect of the extreme due to its fat tailed characteristic. In this paper, we determine appropriate distribution for extreme returns of Nikkei225 Index and analyze the importance of the generalized logistic distribution in modelling extreme events in the financial market in order to accurately conduct risk measure analysis.
\end{abstract}

Keywords: Extreme Value Distribution, Generalized Logistic Distribution, Sub Period Technique, Probability Weighted Moments

\section{INTRODUCTION}

Many financial institutions are faced with high possibilities of risk. These risk arise due to various factors including market performance, portfolio size and failure of internal conducts. Whilst exposed to a variety of risks, the institutions still have an obligation to its clients and must reserve enough capital to sustain these obligations. As such, many regulatory agencies such as the BCBS, Federal Reserve Board and the Federal Deposit Insurance require institutions to have a minimum capital requirement in reserves. This minimum capital is required to act as a cushion when businesses are impacted by large losses and to reduce the chance of running into bankruptcy or default. Institutions therefore make it an important duty to calculate how much of this capital is required to buffer the institution against large losses. Many institutions use the risk measures Value-at-Risk and expected shortfall to quantify these exposures to financial risks and to estimate the necessary capital to protect them against these extreme losses. Since VaR is a quantile risk measure, it is believed to be a good measure for capturing tail extreme events. In the financial literature, it is usually assumed that the daily log returns of indexes follow a normal distribution. Two popular examples of this assumption is the Black-Scholes-Merton (1973) framework which assumes stock prices follow a Geometric Brownian motion in the option pricing model and Sharpe (1964) who assumes normality of the distribution for the stock returns in deriving the capital asset pricing model. However, after extensive research and analysis of this data type, it had been proven to be skewed data. 
Therefore, under the normal assumption, VaR analysis tends to drastically underestimate the true extreme financial losses the institution may be exposed to if the index drastically falls. It is therefore of importance to identify the best distribution that fits the heavy left tail of the financial time series. The determination of adequate model for extreme stock movement was an important problem that thus arose in the financial industry. It is under these circumstances of modelling extreme events in order to conduct risk measure analysis that the concept of Extreme Value Theory was developed.

Extreme Value Theory (EVT) allows one to assess the probabilities and distribution of events that are more extreme than others previously observed. The application of EVT is seen in disciplines that require careful monitoring of extreme events and the casualties they may cause. In finance, EVT focuses only on extreme returns rather than all returns, which is of importance to the institutions and regulators. There have been a variety of work done where EVT was applied to various aspects of the financial sector. [1] Forecast VaR for the long and short positions separately with unconditional and conditional EVT and judge the performance comparing with the other models. [2] Applied EVT to daily returns of crude oil prices in the Canadian spot market from 1998 to 2006. [3] Test the forecasting efficiency of the existing VaR methods for 20 different stock markets and find that the EVT can do the backtesting best. [4] Compare the accuracy of VaR estimation with EVT and GARCH separately employing data from Asian stock markets. [5] Apply univariate EVT to the FTSE100 Index and S\&P 500 indices. Their study shows that EVT performs well in fitting financial market return series for predicting static VaR and ES. [6] study the relation between the Chinese stock market and other 5 stock markets with EVT, their result shows that GL is better for the downside extreme market movements and GEV is better for the upside extreme market movements. [7] Compared the accuracy of GARCH-EVT approach for VaR calculation with other models using data from six stock markets of the world and find that the GARCH-EVT method outperforms in estimating VaR.

In this paper we seek to characterize the distribution of extreme stock returns for the Nikkei225 index from 1995 to 2015. The fatness of the tail of the limiting distribution can be used to calculate the probabilities of a market crash and thus can contribute to the early warning of market risk (Jansen and De Vries, 1991). [8] Applied the two well known methods under EVT, Block Maxima Approach and the Peak over Threshold, to the data which leads to different but closely related descriptions of the extremes. The methods model the extreme events by fitting the Generalized Extreme Value (GEV) distribution and the Generalized Pareto (GP) Distribution, whereafter risk measure analysis was conducted using both models. [8] Concluded that the GP is the better model as there is less wasting of data. However, [9] states that although the GP distribution does have its advantages over the GEV, it is subjected to more serial dependence. [9] Therefore applied a non-overlapping sub period technique to the financial data to reduce dependency. He then fit the Generalized Logistic (GL) distribution and the GEV to the data and compared the results to determine which model better describes the extreme events. [10] Applied GEV and BGEVA models to the sample of Italian SMEs from 2006 to 2011 and found each of the two model is better than the logistic regression. [9] Introduced the option of probability weighted moments as a more efficient way to estimate parameters instead of the maximum likelihood approach, which was the method used in [8]. Each sub period is analyzed and the better fit distribution is assigned to each sub period, where after he concluded that the extremes are generally better characterized by the GL distribution.

The aim of this paper is to apply Extreme Value Theory to financial returns and determine the best distribution which fits the returns. We apply the data sub period technique of [9] on three 
global financial indices and fit the GL and GEV distributions to the data set. We then compare the results from using the sub period technique to [8] who analyzed the fit of the GEV distribution to the entire time horizon. Based on our results, we analyze which methodology is more efficient and sufficient for choosing the adequate distribution that describes the financial market extremes. Finally, we perform VaR analysis given the optimal distribution to determine capital requirement.

Section 2 details the technical content surrounding the different distributions, obtaining model parameters as well as goodness of fit testing. Section 3 provides a description of the data sets used and results obtained. Section 4 covers a critical analysis of the results as well as advantages and drawbacks of both approaches. Finally, we end the paper with our conclusion.

\section{Distributions}

\section{METHODOLOGY}

We make mention of two approaches that model and analyze the behavior of extreme events. These are the Peak over Threshold approach that utilizes the GPD distribution and the Block Maxima Approach which more popularly utilizes the GEV distribution and the more recent introduction of the Generalized Logistic distribution. In figure 1, we observe from the probability distribution density functions of the GPD, GEV and GL that the tails are similar in distribution. As such, the three distributions are asymptotically equivalent in their tails [11]. It is with this equivalence in the tails that we focus our attention on the three distributions to model extreme stock returns.

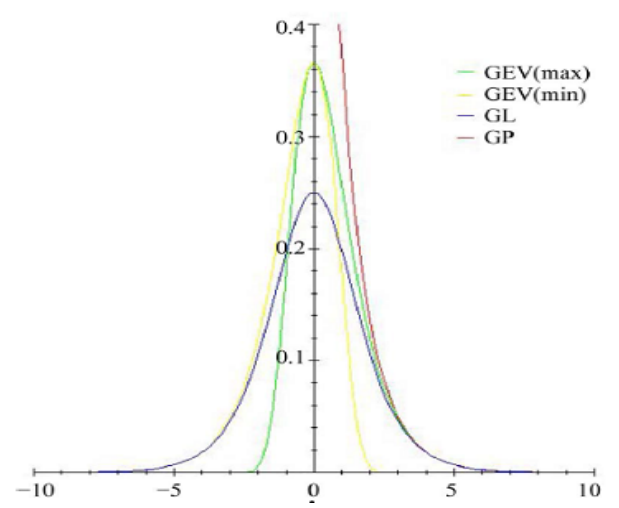

Figure 1: Probability density functions of GEV (max), GEV (min), GP and GL distributions.

The Peak over Threshold Approach models all values that exceed a fixed or high level threshold. This approach follows the Pickands-Balkema-de Haan (1975) theorem. The theorem indicates that the limiting distribution of excesses over a high enough threshold provides a theoretical foundation for us to use the GPD to develop estimators and quantiles. The GPD is argued to be more advantageous than the GEV and GL as it makes use of more data points from the tails. As such, there exists a greater number of available data for the model to fit. However, this approach has two drawbacks: choosing a suitable threshold and being subjected to serial dependence. The choice of a threshold is critical as if it is too high then there are less observations available for accurate estimation and if too low will lead to many central observations in the sample. Additionally, EVT is based on the assumption that the extremes are independent and identically distributed. As financial returns tend to cluster and have high serial dependence, the GP approach is more likely to violate the i.i.d assumption that underlies the EVT concept. Also take into consideration the modelling of extreme events using the sub period technique that is of focus in this paper. This modelling approach is not fit for assuming the GPD distribution. As such, we focus on the GEV and GL distributions. 
The Block maxima approach utilizes the assumption that the log returns are independent and identically distributed. It is realized as a traditional method that groups the returns into nonoverlapping blocks of equal length and models the maxima of each adjoining block with a suitable distribution. The choice of block size is very critical as there exists a bias-variance trade off between the block length and the number of blocks. This traditional approach follows the [12] and Gnedenko (1943) theorem. The theorem indicates that under EVT, the limiting distribution of the extremes collected over non-overlapping time periods of equal length, after being normalized and centered, ought to be one of the three distributions that make up the GEV family (Weibull, Gumbel and Frechet distribution).

If shape parameter is 0 , then it is the Gumbel distribution; if shape parameter is greater than 0 , it is the Weibull distribution and if less than 0, it is Frechet distribution. We take particular note of the shape parameter which governs the shape of the distribution, where larger absolute values of shape parameter imply heavier tailed distributions. [8] And [9] are two of the many works that have proven extreme financial returns generally follow the Frechet distribution, a distribution with a heavy left tail.

However in a number of recent EVT analysis, various authors have empirically shown that the extremes of financial returns can adequately be modelled by the generalized logistic distribution (GL) over the GEV distribution. [13] Studied the distribution of extreme stock market index on both tails and discovered that the GL distribution characterized the extremes better than the GEV and GP for daily, weekly and monthly financial returns as there is persisting evidence of autocorrelation and heteroskedasticty in the financial data. This owes to the fact that the GL distribution has a fatter tail than the other aforementioned distributions. [11] Have also in their research proved the theoretical importance of GL distribution in extreme value modelling: a fatter-tailed distribution is better able to fit large extremities that lie in the tail, reducing underestimation error.

\section{Model Selection}

The selection the distribution that best fits the financial returns is determined by using Lmoment ratio diagrams. L-moments (Hosking 1990) are expectations of certain linear combinations of ordered statistics (L-statistics), used to summarize the shape of a probability distribution. They can be used to calculate mean, standard deviation, skewness and kurtosis.

A theoretical distribution has a set of population L-moments. Sample L-moments can be defined for a sample from the population, and can be used as estimators of the population Lmoments. We identify the distributions that best fit empirical data by plotting the estimated skewness and kurtosis from the data set and choosing the distribution whose theoretical Lskewness and L-kurtosis curve is closest to the observed plotted points.

\section{Parameter Estimation}

Estimating parameters for the models are subjected to sampling errors. Therefore, a method of estimating parameters that minimize these errors must be chosen. Parameter estimates for the limiting distributions are calculated using the probability weighted moments (PWM) technique outlined in [9] and [13] instead of the conventional MLE as used in [8] and other researchers. This technique was chosen as it generates more unbiased parameter estimates than popular MLE method for small sample sizes, which is the norm for EVT data sets. There also exists a linear relationship between PWM and the more robust L-moments as to why this technique was chosen. The PWM method estimates a distribution's parameters by equating the sample moments to those of the fitted distribution. 


\section{Goodness of Fit}

The Anderson-Darling test [14] is used to assess how effective the chosen distribution is to fit the extremes. The test is most often used in contexts where a family of distributions is being tested, in which case the parameters of that family need to be estimated. This goodness of fit test is believed to be the best option in this analysis for measuring the discrepancies in the tails between theoretical and empirical distributions based upon a small sample size [15]. [16,17] describe the AD test as the most powerful statistical tools for detecting how great the sample moves away from normality. The test statistic includes a weight function that places greater emphasis on the tails of the distribution. As such, it is the best model test for heavy tailed distributions.

\section{DATA DESCRIPTIONS AND RESULTS}

This paper focuses on the results of modelling extreme maxima daily returns over weekly intervals. That is, we focus on the maxima extreme returns which occur on the right tail of the empirical distribution observed in each week and determine the best fitting distribution for the weekly maxima data set. These maxima daily returns are collected over non-overlapping successive selection intervals of 5 days. Daily log returns of the Nikkei225 indexes were the underlying data used to assess the effectiveness of the chosen distributions to fit the extreme returns. The closing prices were downloaded for different time periods based on availability of the data. As a result, the historic time periods under analysis are 1995-2015 for the Nikkei225 index.

Figure 2 illustrates the QQ plot of daily log returns against the normal distribution for the Nikkei225. Notice in the diagram that the data does not follow the pattern of the normal distribution, which as previously mentioned is universally assumed. We observe the existence of deviation from the normal in both the left and right tails, justifying the use of the EVT distributions to model the right tail distributions of the three indexes.

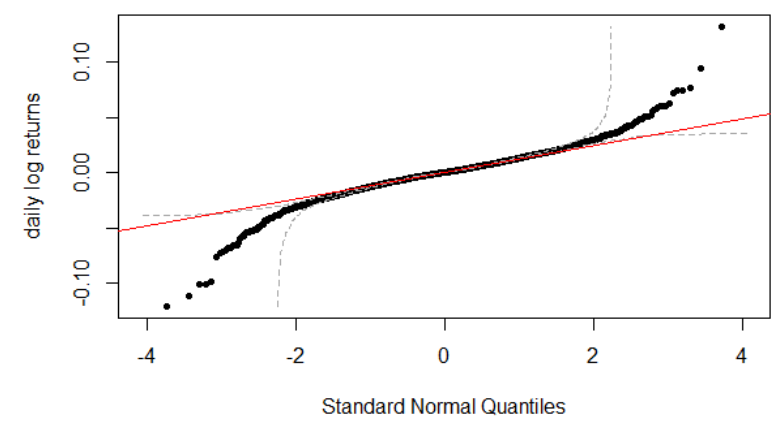

Figure 2: QQ plot of daily log returns against the normal distribution

The time span chosen also contains some key volatile moments in history which have negatively impacted the chosen financial markets, from which proper EVT modelling should capture if sufficient. These extreme historic moments include but are not limited to the 2008 financial crisis, the 2011 Japanese earthquake and the 2001 US terrorist attack. The weekly maxima extremes (right tail extremes) were collected for the Nikkei225 over the period 1995 to 2015. We apply the moving window techniques where the log financial returns are divided into yearly selection intervals each of size 51-52 extremes. As a result, we not only determine the distribution which best fits the entire sample period, but the distribution that fits each of the 21 sub respectively. This technique is argued to reduce the serial dependency of financial returns by capturing the non-stationary of the data. 
The first step in our analysis is to assume probability distributions that are likely to provide good descriptions of the financial series. As previously mentioned, the focus of analysis lies on the GEV and GL distributions. The values of skewness against kurtosis for the series of maxima over each selection interval were estimated and plotted on an L-moment ratio diagram. Figures 3 shows the relationship between sample estimates of skewness against kurtosis and that of the theoretical GEV and GL curve respectively. The diagrams reveal that the samples of the weekly maxima are generally dispersed and fall in the region between the theoretical GEV and GL distributions for the three indexes. Based on this observation, we run further statistical tests on the GEV and GL distributions.

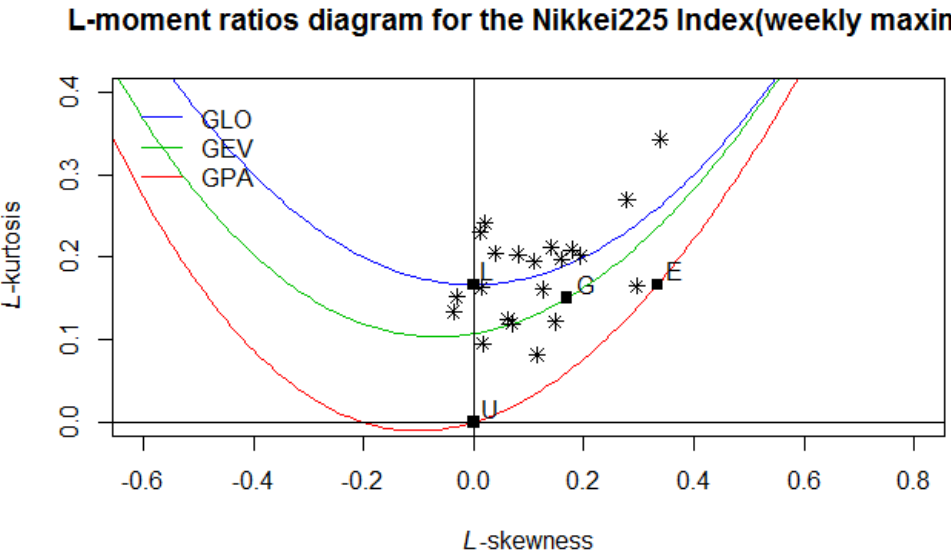

Figure 3: L-moment ratio points for Nikkei225 weekly maxima, divided into 21 sub periods, over 1995 to 2015

The second step is to determine which of the distributions (if any) better fit the extreme returns for each sub period and for the entire sample time horizon as well. The GEV and GL distributions were fitted to the weekly maxima returns for each of the different 21 sub periods for the Nikkei225 index. The parameters for both models were estimated using PWM technique, then the Anderson-Darling goodness of fit test is conducted for model efficiency for the three indexes. The tables for all three indexes illustrate the location, scale and shape parameter estimates for the distributions together with the critical p-values of the AD goodness of fit test.

We observe the results for the Nikkei225 index in table 1 after fitting the GEV distribution and the GL distribution. The shape parameter takes an overall negative value for the entire 21year-period of -0.010 , showing the 21-year weekly maxima returns is modelled by a Frechet distribution. However, notice once more that dividing the time horizon into yearly sub periods for both distributions, the shape parameter fluctuates between negative and positive values. This shows us that different time periods have different skewness of distribution depending on what is going on in the market at that point in time. The Anderson-Darling p-values of 0.0442 and 0.0011 for the GEV and GL distributions respectively are both lower than 0.05 when both distributions were fitted to the entire 21-year-period. This indicates that both distributions provide an inadequate fit to the Nikkei225 index for the whole 21 years. An explanation for the ill-fit of both models is that the nature of the distribution of the extremes was changing over time. Therefore, when the entire 21-year-period was used, the data came from a mixture of distributions, making it di cult for a single distribution to provide a better fit. When the weekly maxima were divided into 21 sub periods, both the GEV and GL provided an adequate fit for 20 of the 21 sub periods. Both the GEV and GL distributions failed to adequately fit the maxima returns in sub period 17 which corresponds to the 2011 Japanese earthquake in North Japan. 
The effect of this earthquake on the Japanese economy caused the index to plummet drastically within this period. Again, we observe that the shape parameter for both distributions takes its maximum values in this sub period. In comparison to each other, the GL provided a better fit for 14 of the 21 sub periods, whilst the GEV fit better for only 7. Unsuspectingly, the average sub period scale parameter exceeded that of the entire sample period for both the GEV and GL.

Table 1: GEV and GL parameters Estimates for NIKKEI 225

\begin{tabular}{|c|c|c|c|c|c|c|c|c|c|}
\hline SUBPERI OD( S) & \multicolumn{2}{|c|}{ LOCATI ON } & \multicolumn{2}{|l|}{ SCALE } & \multicolumn{2}{|l|}{ SHAPE } & \multicolumn{2}{|c|}{ AD P-VALUE } & \multirow[t]{2}{*}{ FI T } \\
\hline & GEV & G & GEV & G & GEV & G & GEV & G & \\
\hline$S=1$ & 0.011 & 0.014 & 0.009 & 0.006 & -0.01 & -0.174 & 0.0442 & 0.0011 & GEV \\
\hline \multicolumn{10}{|l|}{$S=21$} \\
\hline 1 & 0.01 & 0.014 & 0.009 & 0.006 & 0.017 & -0.159 & 0.9263 & 0.9806 & GL \\
\hline 2 & 0.007 & 0.01 & 0.006 & 0.004 & 0.215 & -0.038 & 0.7895 & 0.9886 & GL \\
\hline 3 & 0.013 & 0.017 & 0.008 & 0.006 & -0.187 & -0.296 & 0.8652 & 0.7394 & GEV \\
\hline 4 & 0.012 & 0.016 & 0.01 & 0.007 & -0.036 & -0.194 & 0.9735 & 0.9976 & GL \\
\hline 5 & 0.0113 & 0.015 & 0.0094 & 0.006 & 0.096 & -0.11 & 0.9195 & 0.9825 & GL \\
\hline 6 & 0.011 & 0.014 & 0.008 & 0.005 & 0.071 & -0.125 & 0.8867 & 0.908 & GL \\
\hline 7 & 0.015 & 0.019 & 0.013 & 0.008 & 0.087 & -0.115 & 0.9467 & 0.7748 & GEV \\
\hline 8 & 0.013 & 0.017 & 0.011 & 0.007 & 0.145 & -0.08 & 0.8451 & 0.9706 & GL \\
\hline 9 & 0.014 & 0.016 & 0.008 & 0.004 & 0.259 & -0.014 & 0.9 & 0.9659 & G \\
\hline 10 & 0.01 & 0.012 & 0.008 & 0.004 & 0.339 & 0.037 & 0.9953 & 0.9947 & GEV \\
\hline 11 & 0.009 & 0.012 & 0.007 & 0.004 & 0.176 & -0.062 & 0.9751 & 0.9325 & GEV \\
\hline 12 & 0.01 & 0.012 & 0.006 & 0.004 & 0.034 & -0.148 & 0.9867 & 0.9333 & GEV \\
\hline 13 & 0.01 & 0.014 & 0.011 & 0.006 & 0.248 & -0.02 & 0.3331 & 0.6845 & GL \\
\hline 14 & 0.016 & 0.022 & 0.014 & 0.009 & -0.162 & -0.278 & 0.9286 & 0.9919 & GL \\
\hline 15 & 0.013 & 0.016 & 0.008 & 0.005 & 0.046 & -0.14 & 0.8277 & 0.9594 & G \\
\hline 16 & 0.01 & 0.0142 & 0.012 & 0.007 & 0.262 & -0.012 & 0.9355 & 0.9211 & GL \\
\hline 17 & 0.011 & 0.013 & 0.006 & 0.004 & 0.34 & 0.31 & 0.0001 & 0.0002 & GL \\
\hline 18 & 0.0122 & 0.015 & 0.008 & 0.005 & 0.254 & -0.017 & 0.9908 & 0.9221 & GEV \\
\hline 19 & 0.014 & 0.017 & 0.009 & 0.005 & 0.164 & -0.069 & 0.9435 & 0.8893 & GEV \\
\hline 20 & 0.009 & 0.012 & 0.007 & 0.004 & -0.0122 & -0.178 & 0.9434 & 0.9915 & GL \\
\hline 21 & 0.008 & 0.01 & 0.006 & 0.005 & -0.248 & -0.306 & 0.8218 & 0.9325 & GL \\
\hline
\end{tabular}

The VaR at the 99\% confidence level reveals some of the smallest values in the sub periods that were very volatile and affected those in the long position. Take for example sub period 17 of the Nikkei225 index. The VaR results were $2.6 \%$ and $2.8 \%$ given the GEV and GL distributions respectively. As previously mentioned, this sub period is related to the 2011 Japanese earthquake. Individuals therefore with a short position in stock were less exposed to risks.

Also, we notice that the observed VaR for the entire periods did not exceed the average sub period VaR. This reveals the true purpose of our research as we can conclude that the VaR estimates can be greatly underestimated depending on the length of the sub periods. The same conclusion holds when we compare the VaR results across the two different distributions. We generally observe that the distribution which provided the better fit gave a better VaR estimate than the other. 
Table 2: VaR Estimates via GEV and GL at $99 \%$ confidence level

\begin{tabular}{|c|c|c|c|c|c|c|c|c|}
\hline SUBPERI OD( S) & GEV & GL & SUBPERI OD( S) & GEV & GL & SUBPERI OD( S) & GEV & GL \\
\hline$S=1$ & 0.051 & 0.055 & & & & & & \\
\hline \multicolumn{9}{|l|}{$S=21$} \\
\hline 1 & 0.053 & 0.057 & 8 & 0.05 & 0.054 & 15 & 0.048 & 0.051 \\
\hline 2 & 0.026 & 0.029 & 9 & 0.035 & 0.038 & 16 & 0.041 & 0.045 \\
\hline 3 & 0.074 & 0.078 & 10 & 0.027 & 0.03 & 17 & 0.026 & 0.028 \\
\hline 4 & 0.062 & 0.066 & 11 & 0.031 & 0.034 & 18 & 0.035 & 0.038 \\
\hline 5 & 0.047 & 0.05 & 12 & 0.036 & 0.038 & 19 & 0.043 & 0.046 \\
\hline 6 & 0.04 & 0.043 & 13 & 0.04 & 0.044 & 20 & 0.041 & 0.043 \\
\hline 7 & 0.063 & 0.069 & 14 & 0.11 & 0.115 & 21 & 0.059 & 0.06 \\
\hline
\end{tabular}

\section{CRITICAL ANALYSIS}

Many applications of EVT in finance tend to focus on either the GEV or GP distributions. However, the GL distribution has a fatter tail than the aforementioned distributions. Therefore, it is expected to be a better fit for extreme value and better estimate tail measures such as VaR. The works of Tolikas (2008) and Tolikas and Gettinby (2009) argue that overall the GL distribution proves a better fit when multiple sub periods are used. Hussain (2015) concludes that the GEV distribution is the best distribution to fit the extremes that exist in the right tail of indexes. Gilli and Kellezi (2006) on the other hand identified the GP distribution as the better choice for modelling extreme events in financial markets over the GEV distribution. However, the results revealed that both the GEV and GP distributions are necessary for modelling the extreme maxima returns for the Nikkei225 index. The results revealed that the GL distribution adequately fit more sub periods than the GEV but neither distribution provided an adequate fit to the Nikkei225 maxima returns for the entire 21-year-period. The sign of the shape parameter tends to change over each sub period, indicating no unique distribution can adequately describe the empirical data well.

Additionally, upon dividing the entire time horizon into sup periods, it is observed that the size of the extreme maxima varies constantly over time. This is indicated by the substantial variability observed in the shape parameters over the different sub periods as mentioned above. We observe that those sub periods with extremely large losses had high volatility parameters and in turn resulted in higher shape parameter than those with smaller observable losses. This result would have a great effect on VaR estimates as those periods with higher shape parameters are expected to have greater VaR estimates. Therefore, industries who choose to use shorter period for measuring their exposure to financial risks would have a greater minimum capital requirement than those who choose to conduct analysis on extended time horizons. Therefore there are both advantages and disadvantages to this methodology of obtaining the extreme maxima returns. An advantage of analyzing the extreme losses using the sub period technique is that the VaR estimates are more likely to respond to changes in the market faster than using one extended time horizon. However, a disadvantage is having a larger minimum capital requirement reserve than needed, leading to less available funds to the organization to conduct other business. We can therefore conclude that the choice of distribution and time period to model the behavior of extreme returns has important implications for investors who wish to assess the risk of a portfolio, and for financial regulators who employ VaR based on the distribution.

Financial returns exhibit heteroscedasticity and serial correlation. [9] Argues that the sub period technique reduces the dependency that financial series may be subjected to by selecting maxima extremes from non overlapping blocks of equal length. This approach is similar to the 
block maxima approach but differs in the sense that the best fit distribution is determined for each predetermined sub period and not the entire time horizon. However, for shorter periods, we cannot guarantee the same level of confidence of reduced heteroscedasticity. [18] Suggest modelling heteroscedastic financial time series by fitting the tail of the conditional distribution of returns using an autoregressive volatility model, standardizing the returns by the estimated conditional volatility and finally conducting the EVT analysis. However, this method requires additional parameter estimation which leads to increased possibility of estimation error and model risk. Additionally, further research can be conducted to compare the EVT analysis with the GARCH-based approach in estimating the VaR.

L-moment ratios were used to determine the candidate distributions that could possibly model the extreme financial returns. This method of identifying distributions has a number of advantages we make note of. The main advantage of the L-moments is that they are more robust to the presence of outliers than conventional moments by being linear combinations of the ordered data. This is because conventional moments include powers that give greater weight to outliers that can lead to bias and variance in the estimators. Another advantage of Lmoments is that sample L-moments can take any value that the population moments can take while conventional moments have bounds. Finally, the asymptotic biases of the L-moments are negligible for most distributions. Efficient parameter estimation plays an important role for measuring the financial risks associated with extreme events. Institutions must select the best method for deriving estimates so that parameters are not greatly under/overestimated and yield inaccurate results. The probability weighted moment technique was chosen in order to determine parameter estimates. [19] Argues that the asymptotic properties of MLE are more open to doubt in the case of small samples where convergence of the likelihood function is not always guaranteed to be at the global maximum, MLE is a better fit for moderate and large samples. As we are working with sub periods of 50-53 observations each, probability weighted moments (PWM) was chosen to estimate parameters. PWM tends to have a lower root-mean square error for small sample sizes than MLE. PWM are fast and straightforward to compute and almost always yield feasible values for the estimated parameters [20]. However, one disadvantage of estimating PWMs depends on the choice of plotting positions. Thus careful choice of plotting positions is important.

\section{CONCLUSIONS}

This paper provides insight for risk assessment of extreme events in stock markets. Financial institutions are now more concerned with managing market risks due to the increase in market volatility of recent times. The results above show that the assumption that returns follow a normal distribution is not an adequate assumption to make as the distribution of financial returns tend to generally be skewed. This can lead to substantial underestimation of the extreme risks involved in the financial markets. The extreme value analysis on VaR estimates shows that without analyzing the extreme events that lie on the tail, the VaR is greatly underestimated. The choice of selecting the most appropriate distribution can therefore have serious implications on stock market risk management. Computational errors would mean either having to maintain high minimum capital reserve to remain solvent in the case of overestimation, or facing great losses in the case of underestimation.

We apply the Extreme Value Theory method to the log returns of Nikkei225 stock index in order to derive estimates for VaR. The analysis of the extremes revealed that major estimation error can occur if the best fit distribution to the extremes is not chosen. It was determined that in most instances the GEV provided a better overall fit for the distributions based on recent historical data. However, applying the same distribution to subsets of the entire time horizon indicated that different distributions adequately fit different time periods based on the market 
performance in that period. This leads to the conclusion that no one distribution can adequately model the extreme maxima. Finally, if the type of data set that financial institutions wish to perform tail analysis on is not i.i.d, EVT based analysis does not provide the best results when modelling the extreme events. We therefore advice that practitioners check for dependence in the data before applying EVT.

\section{ABBREVIATIONS}

The following abbreviations are used in this manuscript: Nikkei 225 Index from http://finance.yahoo.com

\section{Reference}

1. Marimoutou, V., Raggad, B., Trabelsi, A. (2009). Extreme Value Theory and Value at Risk: Application to oil market. Energy Economics, 31(4), 519-530.

2. Feng Ren., David E. Giles (2010). Extreme value analysis of daily Canadian crude oil prices. Applied Financial Economics, 20(12).

3. Dimitrakopoulos. Manolis G. Kavussanos (2010). Value at risk models for volatile emerging markets equity portfolios. The Quarterly Review of Economics and Finance, 50(4), 515-526.

4. J. Kittiakarasakun, Y. Tse (2011). Modeling the fat tails in Asian stock markets. International Review of Economics and Finance, 20, 430-440.

5. David E. Allen, Abhay K. Singh, Robert J. Powell (2013). EVT and tail-risk modelling: Evidence from market indices and volatility series. The North American Journal of Economics and Finance, 26, 355-369.

6. Hussain, SI \& Li, S (2009). Modeling the distribution of extreme returns in the Chinese stock market. Journal of International Financial Markets, Institutions and Money, 34(0), 263-276.

7. Madhusudan Karmakar, Girja K. Shukla (2015). Managing extreme risk in some major stock markets: An extreme value approach. International Review of Economics \& Finance, 35, 1-25.

8. Gilli, M. (2006). An Application of Extreme Value Theory for Measuring Financial Risk. Computational Economics, 27(2-3), 207-228

9. Tolikas, K. (2008). Value-at-risk and Extreme Value Distributions for Financial Returns. The Journal of Risk, 10(3), 31

10. R. Calabrese, S.A. Osmetti. (2013). Modelling SME loan defaults as rare events: the generalized extreme value regression model, Journal of Applied Statistics, 40 (6), 1172-1188

11. Nidhin, K., Chandran, C. (2013). Importance of Generalized Logistic Distribution in Extreme Value Modeling. Applied mathematics, 4(03), 560.

12. Fisher, R. and Tippet, L.H.C. (1928). Limiting Forms of the Frequency Distribution of Largest or Smallest Member of a Sample. Proceedings of the Cambridge Philosophical society, 24:180-190

13. Tolikas, K., Gettinby, G. D. (2009). Modeling the Distribution of the Extreme Share Returns in Singapore. Journal of Empirical Finance, 16(2), 254-263.

14. Anderson TW, Darling DA. (1952). Asymptotic theory of certain "goodness-of-fit" criteria based on stochastic processes. Ann Math Stat 23:193-212

15. Choulakian, V., Stephens, M. A. (2001). Goodness-of-Fit Tests for the Generalized Pareto Distribution. Technometrics, 43(4), 478-484.

16. Arshad M, Rasool MT, Ahmad MI. (2003). Anderson Darling and modified Anderson Darling tests for generalized Pareto distribution. Pak J Appl Sci 3(2):85-88

17. Shin, H., Jung, Y., Jeong, C., Heo, J. (2012). Assessment of modified Anderson-Darling test statistics for the generalized extreme value and generalized logistic distributions. Stochastic Environmental Research and Risk Assessment, 26(1), 105-114. 
18. McNeil, A. J., Frey, R. (2000). Estimation of Tail-related Risk Measures for Heteroscedastic Financial Time series: An Extreme Value Approach. Journal of empirical finance, 7(3), 271-300.

19. Hill, B. M. (1975). A Simple General Approach to Inference about the Tail of a Distribution. The annals of statistics, 3(5), 1163-1174.

20. Hosking, J. R. M., Wallis, J. R., \& Wood, E. F. (1985). Estimation of the Generalized Extreme-Value Distribution by the Method of Probability-Weighted Moments. Technometrics, 27(3), 251-261. 